\title{
Global school-based student health survey: country profiles and survey results in the eastern Mediterranean region countries
}

\author{
Elham Abdalmaleki', Zhaleh Abdi', Sahand Riazi Isfahani', Sara Safarpoor', Bahar Haghdoost 1,2, \\ Saharnaz Sazgarnejad ${ }^{1,2}$ and Elham Ahmadnezhad ${ }^{1 *}$
}

\begin{abstract}
Background: The increasing prevalence of non-communicable diseases (NCDs) has some major implications on many countries to achieve universal health coverage. This study aimed to investigate the implementation of Global School-based Student Health Survey (GSHS), which is used to assess the risk factors of NCDs among children and adolescents in the eastern Mediterranean region (EMR).

Methods: This study was a meta-analysis and systematic literature review of 2001-2018 published studies, which were found by searching PubMed, Google Scholar, WHO, and CDC databases. In this study, the target group was students aged between 13 and 17 years old. GSHS implementation as well as risk factors of NCDs were compared across different countries. The random-effect model for meta-analysis was considered at 95\% confidence interval.

Result: In the EMR, 19 countries have implemented GSHS at least once following the survey manual (37 surveys). Overall, 201,795 students were included in our analysis. The overall estimation prevalence rateof the overweight was $24.5 \%$ (20.6-28.8), obesity was 7.3\% (5.4-9.5), insufficient physical activity was $82.4 \%$ (80.7-84.1), tobacco usage was $14.3 \%$ (10.53-18.67), and smoking was $9.6 \%$ (8.1-11.3), respectively. Among those aged 13 to 17 years old, these rates were estimated as 19.8 (13.2-27.3), 9.7 (6.2-14.0), 86.1 (84.1-87.9), 17.8 (11.8-24.7), and 11.5 (9.4-13.8), respectively.
\end{abstract}

Conclusion: GSHS has been widely implemented across EMR countries. Using nationally representative data, the results show that more efforts are needed to target the NCDs risk factors among adolescents in the region.

Keywords: Global school-based student health survey, Adolescence, Eastern Mediterranean region, Sustainable development goals, Universal health coverage, Non-communicable diseases

\section{Background}

Nearly $35 \%$ of the global burden of diseases originates in adolescence, and more than 3000 adolescents die every day, mostly due to Non-communicable diseases (NCDs), injuries, and other preventable causes [1]. The Eastern Mediterranean Region (EMR) suffers from

*Correspondence: ahmadnezhad@tums.ac.ir

${ }^{1}$ National Institute of Health Research (NIHR), Tehran University

of Medical Sciences, Tehran, Iran

Full list of author information is available at the end of the article high prevalence rates of NCD-related risk factors [2]. The World Health Organization (WHO) in collaboration with other UN agencies and technical assistance of the Centers for Disease Control and Prevention (CDC) introduced Global School-based Student Health Survey (GSHS) in 2001, which has been started around the world since 2003. The purpose of GSHS is providing accurate data on health behaviors and protective factors among students, in order to help countries develop more priorities; establish appropriate programs; advocate for resources for school health programs and policies; 
and allow international agencies and countries to make comparisons across countries [3]. The GSHS is a rapid, affordable, useful tool and a suitable alternative for surveillance systems $[4,5]$. Of note, the GSHS is a part of the WHO STEPwise approach to Surveillance [4]. Correspondingly, this approach has three steps for data collection, including performing the questionnaire-based assessment, biochemical measurements, and laboratory measurements [6]. In this regard, it is recommended to conduct GSHS every 3 to 5 years [7]. This survey uses a standardized scientific sample selection process, the common school-based methodology, core questionnaire modules, and three questionnaires (including co core questionnaire modules, core-expanded questions, and country-specific questions, which are combined to form a country-specific self-administered questionnaire) [8]. Accordingly, the 10 core questionnaire modules address the leading causes of morbidity and mortality, including alcohol abuse, dietary behaviors, drug abuse, hygiene, mental health status, physical activity, protective factors, sexual behaviors, tobacco usage, violence, and unintentional injury, among children and adults worldwide. Countries must select at least six of the 10 core modules in their country-specific questionnaire $[3,9]$. Thereafter, country-specific questions can be added to the GSHS core questionnaire [10]. About, 99 countries have implemented this survey [11]. The GSHS results could help in guiding policy makers to plan, administer, and implement some interventions targeting the NCD risk factors in adolescents [12]. Following the introduction of the Sustainable Development Goals (SDG) in 2015, monitoring the interventions to reduce NCDs and the related risk factors globally received increasing attention [13, 14]. Therefore, the appropriate implementation of such surveys is of great importance [15]. This study aimed at examining the implementation status of GSHS in the EMR, comparing prevalence rates of NCD risk factors, including alcohol and tobacco abuse, obesity and overweight (inappropriate dietary behaviors), and physical activity among EMR countries as well as estimating an overall value for each one of these indicators in adolescents aged between 13 and 15 and between 13 and 17 years old, using the metaanalysis method.

\section{Methods}

This study was a systematic review and meta-analysis conducted in two consecutive phases. The first phase was the comparison of the surveys' designs, including questionnaires, modules, tools, and the implementation period. In addition, the second phase was the comparison of the results of these surveys, including the indicators for tobacco usage (smoking and consumption of any type of tobacco), alcohol use, obesity and overweight (inappropriate nutritional behaviors), and physical activity in terms of gender in 22 EMR countries. Afterward, the results obtained for each indicator were combined to estimate an overall value for all member states using a meta-analysis design. Prior to 2012, GSHS was only performed among students aged between 13 and 15 years old, but since 2013, the age of the target group has changed to people aged between 13 and 17 years old. Accordingly, these two age groups were compared among EMR countries. For the countries that implemented GSHS once before and once after 2013, the results were presented for both age groups of 13-15 and 13-17 years old. However, the ages of the participants investigated in the studies performed in Iran and Saudi-Arabia were different from those of other countries in other studies. Accordingly, to preserve the consistency, their indicators were considered in the range of 13 to 17 years old.

\section{Phase 1-comparison of surveys conducted across the EMR countries}

The WHO and CDC databases were the main information sources for this study. In cases where there was no data from member states published on these databases, other databases such as PubMed and Google Scholar, were searched. Keywords used for this search included: "Global School Health Survey (GSHS)", "Non-communicable disease Prevention", "school-based survey", "Childhood and Adolescence Surveillance", "Protocol", and "methodology" along with the name of the countries with different combinations of search terms in the subjects and abstracts. Figure 1 shows PRISMA flow diagram, summarizing the review process [1]. Of note, the published surveys reports were not included in the process.

The surveys were compared in terms of the modules, their sample sizes, and response rate using published country reports and factsheets between 2001 and 2018 by the WHO [11]. Information of Iran and Saudi-Arabia surveys were extracted from the published articles. Moreover, the questionnaires and modules were compared across the EMR countries. Finally, core modules and their frequencies of execution were compared across all these included surveys.

\section{Phase 2-comparison of the surveys' results}

The WHO dataset was used as the principal data source to examine the survey results for each country. The indicators of the Saudi-Arabia and Iran studies were extracted from both articles and national reports. The survey's results were combined using a random-effect model of meta-analysis. Thereafter, the statistical heterogeneity was examined using $\mathrm{I}^{2}$ statistic and the statistical significance level was considered as $P$-value $<0.05$. Due to the high heterogeneity among the studies, the 


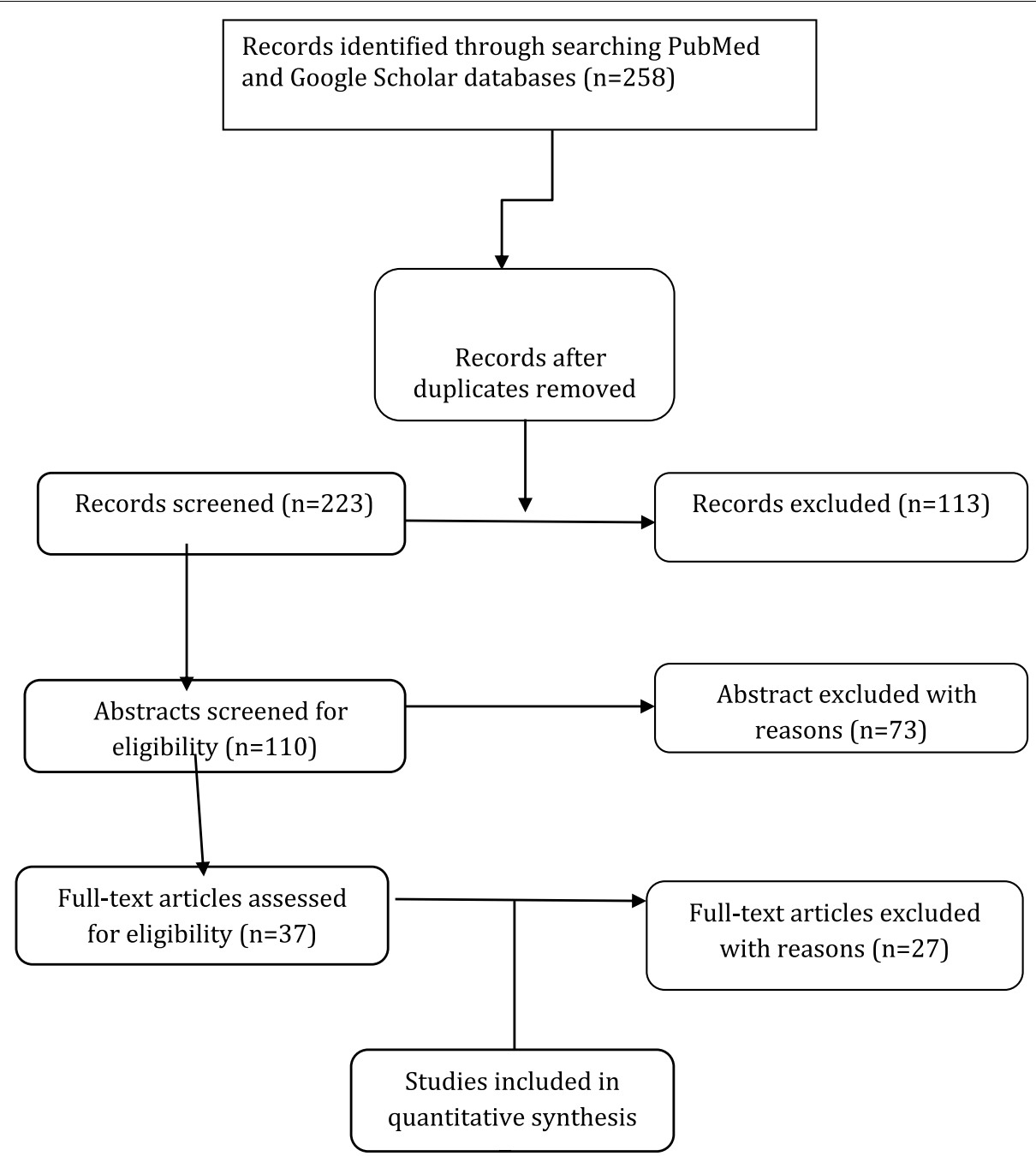

Fig. 1 PRISMA Flowchart. The PRISMA flow diagram for the review detailing the database searches, the number of abstracts screened, and the full texts retrieved

random-effect model was used to combine the results at $95 \%$ confidence interval. For performing statistical analyses, the variance of each study was initially calculated using binomial distribution variance, by considering the binomial distribution of the prevalence rate. The weight given to each study was proportional to the inverse-variance. The included studies were combined in terms of the number of samples and variance. All these analyses were performed using metaprop command in STATA16 software. The cut-off points of each indicator were considered according to the definition of low, medium, and high risks [16]. The definitions for each indicator as well as their cut-off points are explained in the following:

Current tobacco use
- Percentage of students who are currently smoking (smoked cigarettes on at least 1 day during the 30 days before the survey)

- Percentage of students who are currently using any tobacco products (used any tobacco products on at least 1 day during the 30 days before the survey)

- Risk Scoring: < 7 (Low); 7-15 (Medium); $\geq 16$ (High)

Current alcohol use

- Percentage of students who are currently abusing alcohol (at least one drink of alcohol for at least one day during the 30 days before the survey)

- Risk Scoring: <20 (Low); 20-39 (Medium); $\geq 40$ (High) 
Overweight or obesity

- Percentage of students who were overweight $(>+1$ SD from median for BMI by age and sex)

- Percentage of students who were obese $(>+2 \mathrm{SD}$ from median for BMI by age and sex)

- Risk Scoring: <10 (Low); 10-19 (Medium); $\geq 20$ (High)

Physical activity

- Percentage of students who were physically active for at least $30 / 60$ min per day on $5 / 7$ days during the 7 days before the survey

Physical inactivity

- Percentage of not engaging students who were physically active for at least $30 / 60$ min per day on 5/7 days during the 7 days before the survey

- Risk Scoring: <50 (Low); 50-69 (Medium); $\geq 70$ (High)

\section{Results}

\section{Phase 1-comparison of the surveys}

All EMR countries, except Somalia, Iran, and SaudiArabia implemented at least one round of GSHS. In this region, out of 22 (86\%) countries, 19 implemented GSHS once following the international protocol during 20012018. The questionnaires used were those suggested by the international guidelines. The surveys implemented in Iran and Saudi-Arabia, which were a part of the national health surveillance system, were modified versions of the GSHS. In Iran, this modified survey, called CASPIAN study, was implemented in five rounds from 2004 to 2016 [17-25]. In Saudi-Arabia, one round of Jeeluna Study was conducted in 2011-2012 [26]. The age of the target group of these two studies differed from those of GSHSs implemented in other EMR countries (Saudi-Arabia: between 10 and 19 years and Iran: between 6 and18 years). No results were found for the implementation of any international or national study in Somalia. Table 1 shows the survey modules examined in EMR countries. Additional file 1: Appendix 1 represents the list of available publications on GSHS for each EMR country.

Table 2 shows the included surveys' characteristics, including year(s) of implementation, sample size, and response rate percentage. The maximum sample sizes were those of Iran (21,111 students in 2004) and the United Arab Emirates (15,790 students in 2005). In addition, the minimum sample size belonged to Yemen in 2008 (1175 students). The highest and lowest response
Table 1 Surveys' modules examined in EMR countries Table

\begin{tabular}{ll}
\hline No & Module \\
\hline 36 & Dietary behaviors and overweight \\
27 & Alcohol and other drug use \\
37 & Hygiene \\
32 & Mental health \\
35 & Physical activity \\
37 & Protective factors \\
31 & Tobacco use \\
35 & Violence and unintentional injury \\
1 & Sexual behaviours that contribute to HIV infections \\
1 & Other Sexually transmitted infections (STIs) \\
1 & Unintended pregnancies \\
\hline
\end{tabular}

rates were for Jordan (99.8\%) in 2007 and Saudi-Arabia $(62.25 \%)$ in 2012 , respectively.

\section{Phase 2-comparison of the surveys' results}

Table 3 presents the risk levels of the non-communicable diseases' risk factors.

\section{Tobacco use}

Risk levels for tobacco usage were define as follows:

$16 \%$ or above 7-15\% Below 7\%

As shown in Table 3, male adolescents aged between 13- and 15-years old smoke cigarettes or any type of tobacco products at higher rates compared to the female ones at the same age. The highest prevalence rate of cigarette smoking in this age group among males was $25 \%$ in Qatar (2011) followed by Palestine (24\% in 2010). The lowest prevalence rate of smoking cigarettes at this age group was reported as approximately $1 \%$ in females in Libya (2007), Morocco (2006), and Egypt (2011). The highest prevalence rates of using any type of tobacco products in this age group were seen in male adolescents living in Lebanon (2017) and Kuwait (2015), as 35 and $29 \%$, respectively. As well, the lowest prevalence rate was $3 \%$ among females in Tunisia (2007), Libya. (2007), and Morocco (2006).

The prevalence rate of total tobacco usage (cigarettes and any type of tobacco products) among students aged between 13 and 15 years old ranged was 18\% in Qatar (2011) and 3\% in Morocco (2003). The highest prevalence rate for smoking any type of tobacco products in this age group was $31.5 \%$ in Lebanon (2017) and the lowest was 6\% in Libya (2007) and Morocco (2006). 
Table 2 Characteristics of the included surveys

\begin{tabular}{|c|c|c|c|c|c|}
\hline Country & & Survey's Name & Implementation Year & $\begin{array}{l}\text { Sample Size } \\
\text { (Students) }\end{array}$ & $\begin{array}{l}\text { Reponses } \\
\text { Rate (\%) }\end{array}$ \\
\hline \multirow[t]{2}{*}{ Jordan } & & GSHS & 2004 & 2457 & 95.0 \\
\hline & & & 2007 & 2197 & 99.8 \\
\hline Afghanistan & & GSHS & 2014 & 2579 & 79.0 \\
\hline \multirow[t]{5}{*}{ Iran } & & CASPIAN & 2004 & 21,111 & - \\
\hline & & & 2007 & 9171 & - \\
\hline & & & 2010 & 5570 & - \\
\hline & & & 2013 & 13,486 & 90.6 \\
\hline & & & 2016 & 14,274 & 99.0 \\
\hline \multirow{3}{*}{\multicolumn{2}{|c|}{ United Arab Emirates }} & GSHS & 2005 & 15,790 & 89.0 \\
\hline & & & 2010 & 2581 & 91.0 \\
\hline & & & 2016 & 5849 & 80.0 \\
\hline Bahrain & & GSHS & 2016 & 7141 & 89.0 \\
\hline Pakistan & & GSHS & 2009 & 5192 & 76.0 \\
\hline Tunisia & & GSHS & 2008 & 2870 & 83.0 \\
\hline Djibouti & & GSHS & 2007 & 1777 & 83.0 \\
\hline Sudan & & GSHS & 2012 & 2211 & 77.0 \\
\hline Syrian Arab Republic & & GSHS & 2010 & 3102 & 97.0 \\
\hline Iraq & & GSHS & 2012 & 2038 & 88.0 \\
\hline Saudi Arabia & & Jeeluna & 2012 & 12,121 & 62.2 \\
\hline \multirow[t]{3}{*}{ Oman } & & GSHS & 2005 & 2979 & 97.0 \\
\hline & & & 2010 & 1606 & 89.0 \\
\hline & & & 2015 & 3468 & 92.0 \\
\hline Qatar & & GSHS & 2011 & 2021 & 87.0 \\
\hline \multirow[t]{2}{*}{ Kuwait } & & GSHS & 2011 & 2672 & 85.0 \\
\hline & & & 2015 & 3637 & 78.0 \\
\hline \multirow[t]{6}{*}{ Palestine } & Gaza & GSHS & 2010 & 2677 & 95.0 \\
\hline & UNWRA ${ }^{a}$ Gaza & & & 2122 & 95.0 \\
\hline & $\begin{array}{l}\text { UNWRA } \\
\text { Lebanon }\end{array}$ & & & 2187 & 96.0 \\
\hline & $\begin{array}{l}\text { UNWRA } \\
\text { Syrian }\end{array}$ & & & 2120 & 94.0 \\
\hline & $\begin{array}{l}\text { UNWRA } \\
\text { West Bank }\end{array}$ & & & 2015 & 90.0 \\
\hline & West Bank & & & 1908 & 94.0 \\
\hline \multirow[t]{3}{*}{ Lebanon } & & GSHS & 2005 & 5115 & 88.0 \\
\hline & & & 2011 & 2286 & 87.0 \\
\hline & & & 2017 & 5708 & 82.0 \\
\hline Libya & & GSHS & 2007 & 2242 & 98.0 \\
\hline \multirow[t]{3}{*}{ Morocco } & & GSHS & 2006 & 2670 & 84.0 \\
\hline & & & 2010 & 2924 & 92.0 \\
\hline & & & 2016 & 6745 & 91.0 \\
\hline \multirow[t]{2}{*}{ Egypt } & & GSHS & 2006 & 5249 & 87.0 \\
\hline & & & 2011 & 2568 & 85.0 \\
\hline \multirow[t]{2}{*}{ Yemen } & & GSHS & 2008 & 1175 & 82.0 \\
\hline & & & 2014 & 2655 & 75.0 \\
\hline
\end{tabular}

a United Nations Relief and Works Agency for Palestine Refugees (UNRWA) 
Table 3 Risk levels of the non-communicable diseases' risk factors among students aged 13-15 years in EMR counties (percent)

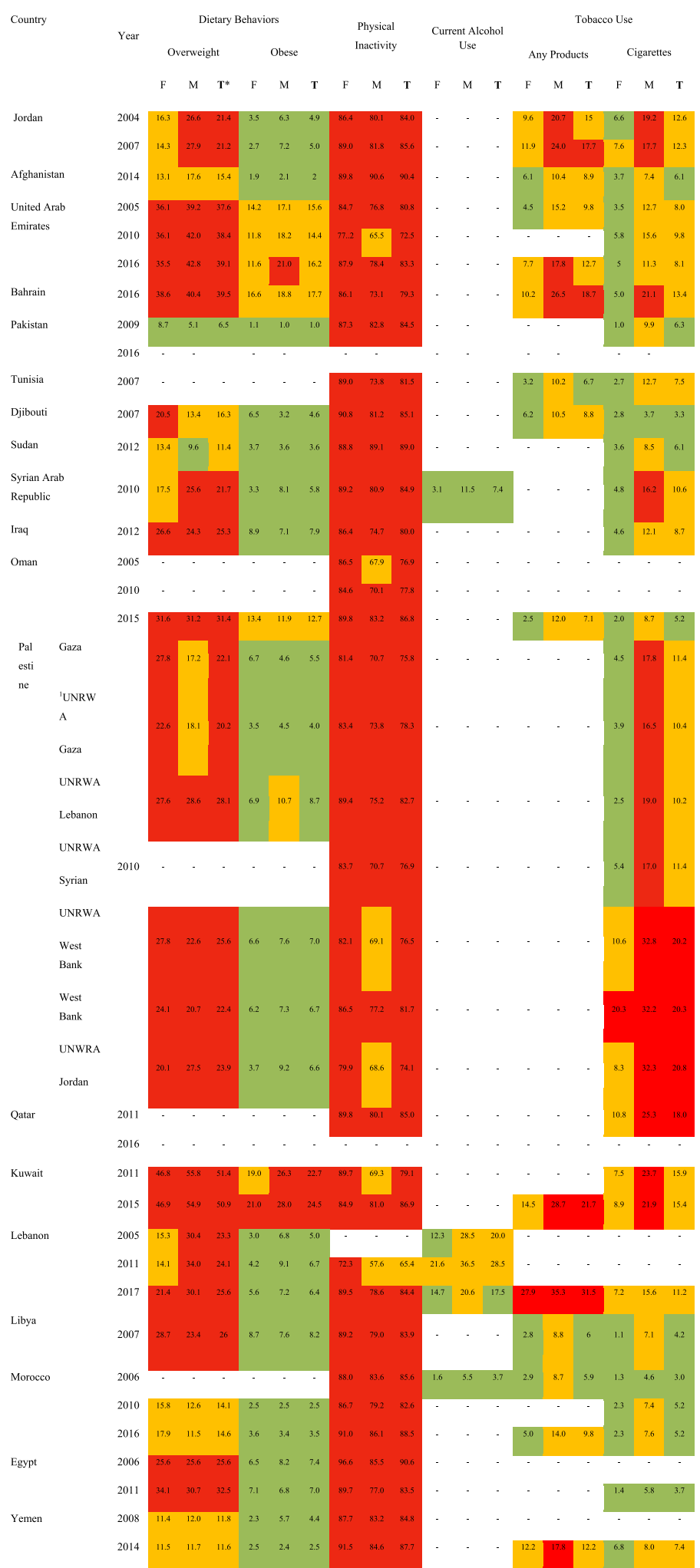

${ }^{1}$ United Nations Relief and Works Agency for Palestine Refugees (UNRWA) 


\section{Physical activity}

Risk levels for physical inactivity were defined as follows:

$70 \%$ or above

$50-69 \%$

Below 50\%

The results shown in Table 3 indicated that the prevalence of physical activity is relatively low among adolescents in the EMR region.

Inappropriate dietary behaviors (obesity and overweight) Risk levels for both obesity and overweight were defined as follows:

$20 \%$ or above 10-19\% Below 10\%

As shown in Table 3, the prevalence of overweight is relatively high in both female and male adolescents. The highest prevalence rates of overweight and obesity were reported in males in Kuwait (56 and 28\%, respectively) and the lowest levels were reported in Pakistan (5 and $1 \%$, respectively). The overall prevalence of overweight, obesity, and physical inactivity were observed as follows: Kuwait has the highest and Pakistan has the lowest prevalence rates of overweight. The prevalence rate of obesity ranged from $1 \%$ in Pakistan to $23.6 \%$ in Kuwait. The highest prevalence of physical inactivity was reported in Egypt (90.6\%) and Afghanistan (90.4\%) respectively. Of note, the lowest prevalence rate was observed in Lebanon (65.4\%).

\section{Alcohol consumption}

Risk levels for alcohol consumption were defined as follows:

40\% or above $\quad$ 20-39\% $\quad$ Below 20\%

The prevalence of alcohol consumption was reported only for three countries, including Lebanon, Morocco, and Syria. In all these three countries, alcohol consumption was higher among men.

As Table 4 shows, among the students aged between 13 and 17 years old, the highest and lowest overweight prevalence rates were reported in Kuwait $(52.9 \%)$ and Iran (4.3\%). In addition, the highest and lowest obesity prevalence rates were reported in Kuwait (28.2\%) and Yemen (1.7\%), among male adolescents, respectively. The prevalence of physical activity was relatively low among adolescents aged 13-17years in the region. The highest and lowest physical inactivity prevalence rates in this age group were in Afghanistan (90.7\%) and Bahrain (80.5\%), respectively. The highest prevalence of cigarettes smokers and any type of tobacco products in this age group was in
Kuwait (28.2\%) and Lebanon (40.9\%), among male adolescents, respectively.

In this study, 37 reports and 10 articles were selected and included to obtain an overall estimation for each indicator by the meta-analysis. The total number of the study participants was 201,795 . The heterogeneity statistics for all the indicators was obtained as approximately $\mathrm{I}^{2}>98$ by combining the results and calculating the overall estimation using a random effect model. Accordingly, this indicated that the results of the studies were extremely heterogeneous. Table 5 shows the total prevalence of overweight, obesity, physical inactivity, and tobacco use (any products and cigarettes) by the two age groups in the EMR. Sex subgroup level analysis was also performed to reduce the existing heterogeneity in results and the heterogeneous results were reported to be high yet (nearly $\mathrm{I}^{2}>99$ ).

\section{Discussion}

This study aimed at examining the implementation status of GSHS in the EMR, comparing prevalence rates of the NCD risk factors, including, alcohol and tobacco use, obesity and overweight (inappropriate dietary behaviors), and physical activity among EMR countries as well as estimating an overall value for each indicator among adolescents aged between 13 and 15 and between 13 and 17 years old.

GSHS has been performed widely across EMR. However, the implemented surveys considerably differed in terms of sample sizes. The CASPIAN study conducted in Iran reported the highest sample size among the other implemented surveys. This review showed that EMR countries have not implemented GSHS in a harmonized manner, which can make it difficult to compare the relative indicators among member states over time [5]. Of note, most of the internationally recommended modules were considered by implemented surveys. However, some subjects such as high-risk sexual behaviors, alcohol and drug use, and reproductive health were not considered in the included studies, mainly due to the cultural factors. Therefore, their prevalence rates were not reported in this study.

The results for the two age groups of between 13 and 15 and between 13 and 17 years old are separately presented in the present study. The prevalence rate of physical inactivity in both age groups was estimated to be more than $80 \%(P<0.001)$. In addition, the risk level was high in most years. The lowest prevalence rate was reported among adolescents aged between 13- and 17-years old living in Afghanistan in 2014. In all the countries, except Sudan and Afghanistan, the prevalence of physical inactivity was higher in male adolescents in comparison to that of female ones. The prevalence rate of overweight in 
Table 4 Risk levels of the non-communicable diseases' risk factors among students aged 13-17years in EMR counties (percent)

(13-17 years old)

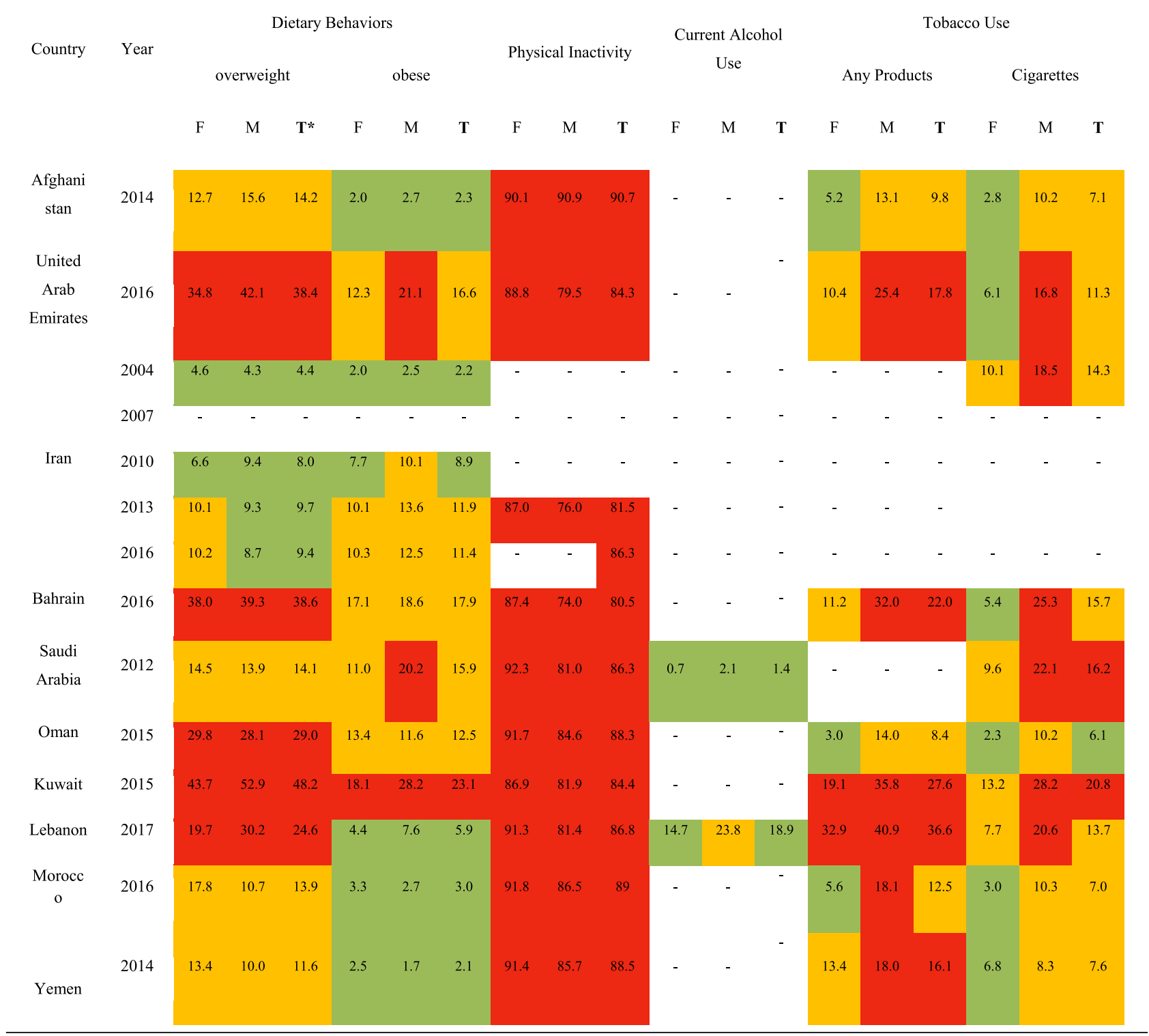

${ }^{*}$ TTotal, $M$ Male, F Female

the region in both age groups was high as well (24.5 and $19.8 \%$, respectively). The results indicate that countries in where students are more physically inactive are more likely to have higher prevalence of overweight and obesity. Nowadays, more than $80 \%$ of the world's adolescent population is insufficiently physically active [27]. Policies to reduce insufficient physical activity are being implemented in only $56 \%$ of WHO Member States in 2013 [28, 29]. Moreover, similar to other countries in the world, the EMR countries had no considerable progress. In this regard, a reduction of at least $10 \%$ in physical inactivity is recommended up to 2025 [28, 30]. Additionally, because of its high prevalence, it should be regarded as a high priority public health problem in EMR countries. Some factors that may contribute to the high prevalence of this factor in the EMR are the followings: lack of access to recreational facilities, lack of outdoor spaces (like parks), inappropriate public transportation, and social and cultural norms in the region. Females in some countries of the region mostly face some personal and social barriers to be engaged in physical activities. Therefore, it is highly recommended to consider country-specific sociocultural 
Table 5 Result of meta-analysis and heterogeneity on prevalence of overweight, obesity, physical inactivity, tobacco use (any products \& cigarettes) by age group (13-15 \& 13-17years old)

\begin{tabular}{|c|c|c|c|c|c|c|}
\hline \multicolumn{7}{|c|}{ Overall (all EMR countries) } \\
\hline \multirow[t]{2}{*}{ Age group } & \multirow[t]{2}{*}{ Result } & \multicolumn{2}{|c|}{ Dietary Behaviors } & \multirow[t]{2}{*}{ Physical Inactivity } & \multicolumn{2}{|l|}{ Tobacco Use } \\
\hline & & overweight & obese & & Any Products & Cigarettes \\
\hline \multirow[t]{3}{*}{$13-15$} & $\begin{array}{l}\text { pooled estimate prevalence } \\
(95 \% \mathrm{Cl})\end{array}$ & $\begin{array}{l}24.5 \\
(20.6-28.8)\end{array}$ & $\begin{array}{l}7.3 \\
(5.4-9.5)\end{array}$ & $\begin{array}{l}82.4 \\
(80.7-84.1)\end{array}$ & $\begin{array}{l}14.3 \\
(10.53-18.67)\end{array}$ & $\begin{array}{l}9.6 \\
(8.1-11.3)\end{array}$ \\
\hline & $1^{2}$ & 99.6 & 99.4 & 98.3 & 99.5 & 98.7 \\
\hline & Pvalue & $P<0.001$ & & & & \\
\hline \multirow[t]{3}{*}{$13-17$} & $\begin{array}{l}\text { pooled estimate prevalence } \\
(95 \% \mathrm{Cl})\end{array}$ & $\begin{array}{l}19.8 \\
(13.2-27.3)\end{array}$ & $\begin{array}{l}9.7 \\
(6.2-14.0)\end{array}$ & $\begin{array}{l}86.1 \\
(84.1-87.9)\end{array}$ & $\begin{array}{l}17.8 \\
(11.8-24.7)\end{array}$ & $\begin{array}{l}11.5 \\
(9.41-13.89\end{array}$ \\
\hline & $1^{2}$ & 99.8 & 99.7 & 98.3 & 99.6 & 99.0 \\
\hline & P-value ${ }^{*}$ & $P<0.001$ & & & & \\
\hline
\end{tabular}

${ }^{*} p$-value for Chi2 statistic for heterogeneity

and environmental factors affecting physical activity in the design of appropriate interventions. Our results indicate that the higher prevalence of overweight among females is most likely due to their lower physical activity compared to males. The highest and lowest prevalence rates of overweight and obesity were reported in Kuwait and Pakistan, respectively. In another systematic review, the physical inactivity status among Arabic-speaking countries was examined in 2018, its prevalence was reported to be very high, which is similar to our results [31]. It was shown that school-based interventions can have important potentials for both obesity prevention and physical activity promotion among school-aged children and adolescents. In this regard, strategies proposed for promoting behavioral change need to take the strong role of the family supports into the account.

In the EMR, the prevalence of tobacco use was higher in male adolescents than female. The highest and lowest prevalence rates were reported in Lebanon (31.5\%) and Morocco (3\%) respectively. Furthermore, the overall prevalence of tobacco usage in the region was reported as $14.3 \%$ among $13-15$ years old age group and as $17.8 \%$ among 13-17 years old age group. Similar to the other parts of the world, rates of tobacco usage among adolescents and young people are increasing in the EMR region as well [32]. Resolution EM / RC64 / 5, was adopted by the 61st session of the Regional Committee for the Eastern Mediterranean in 2017, which urged member states to implement the effective interventions on the prevention and control of the non-communicable diseases [33].

It may be difficult to achieve all SDG 3 targets in the EMR up to 2030. The high prevalence of the risk factors of NCDs among adolescents in this region demonstrated that interventions aiming at reducing risk factors associated with NCDs, have not achieved the expected results so far. It is highly recommended to include culturally relevant, evidence-based trainings in the school curricula. Correspondingly, this could be considered as one of the best opportunities to correct the lifestyle of this age group. As well, it is necessary to provide sufficient facilities for the purpose of improving the physical activity level of young people as well as promoting healthy eating behaviors in schools. This issue should be considered when the allocation of the educational budgets in this region.

To prioritize the interventions needed to tackle the NCDs risk factors based on our results, physical inactivity was found as the most important modifiable risk factor that should be addressed by EMR countries in the near future. Considering the high prevalence of youth smoking in the EMR, it is suggested that tobacco usage in adolescents and its contributing factors be explored further in the region. A coalition of youth advocates for health in the EMR was launched in 2017 and the issue needs to be emphasized in future meetings of the coalition.

Our analysis has some important limitations. First, the quality of secondary data from surveys can affect the final results. Second, the age ranges of the countries surveys vary that should be taken into account while interpreting the results.

\section{Conclusion}

Since implementation of GSHS survey received considerable attentions of EMR member states, it can be concluded that the risk factors of NCDs in the adolescents are reasonably monitored in the region. As a further step, a harmonized survey could be designed in the region, in order to facilitate the comparison of the results across the countries. Although the NCD risk factors are being 
monitored through GSHS surveys, many targets related to the risk factors have not been achieved in the region. It is critical that comprehensive policies be further designed to address adolescent health issues and to measure the impact of any intervention. Furthermore, an appropriate surveillance system should be designed to monitor the current situation of the NCD risk factors among young people. However, survey's implementation per se cannot guarantee the proper monitoring of the NCD risk factors. Further the capacity of EMR countries for surveillance of NCDs, their risk factors and determinants, as well as utilizing the obtained results in NCD programs' monitoring and evaluation should be strengthened. Among the examined risk factors in this study, insufficient physical activity in adolescents, which needs an instant action to prevent and control, should be promptly considered as a major public health problem by all countries.

\section{Abbreviations}

NCDs: Non-communicable diseases; GSHS: Global School-based Student Health Survey; EMR: Eastern Mediterranean Region; WHO: The World Health Organization; CDC: Centers for Disease Control and Prevention.

\section{Supplementary Information}

The online version contains supplementary material available at https://doi. org/10.1186/s12889-022-12502-8.

\section{Additional file 1: Appendix 1.}

\section{Acknowledgments}

The authors would like to thank the anonymous reviewers for their insightful suggestions and careful reading of the manuscript.

\section{Authors' contributions}

$E A, E A b$, and ZhA conceived the paper. EA, SR, and EAb, conducted the statistical analysis and drafted the manuscript. EA, BH, SHS, and ZhA reviewed and edited the manuscript. SS coordinated the overall process. All authors reviewed the manuscript, provided edits, and approved the final manuscript.

\section{Funding}

This research did not receive any specific grant from funding agencies in the public, commercial, or not-for-profit sectors.

\section{Availability of data and materials}

The datasets generated and/or analyzed during the current study are not publicly available due to they don't not provide more necessary information, but are available from the corresponding author on reasonable request.

\section{Declarations}

Ethics approval and consent to participate Not applicable.

\section{Consent for publication}

Not applicable.

\section{Competing interests}

The authors declare that they have no competing interests.

\section{Author details}

${ }^{1}$ National Institute of Health Research (NIHR), Tehran University of Medical Sciences, Tehran, Iran. ${ }^{2}$ School of Medicine, Tehran University of Medical Sciences, Tehran, Iran.

Received: 21 September 2021 Accepted: 3 January 2022

Published online: 19 January 2022

\section{References}

1. World Health Organziation. Noncommunicable Disease Prevention and Adolescents. 2017. Available from: https://plan-uk.org/file/ncd-preve ntion-and-adolescents-report/download?token=tkG9kOxg. Accessed 30 Aug 2021.

2. Fikri M, Hammerich A. Scaling up action on the prevention and control of noncommunicable diseases in the WHO eastern Mediterranean region. East Mediterr Health J. 2018:24(1):3-4.

3. World Health Organization. Global School-based Student Health Survey (GSHS). 2018. Available from: https://www.who.int/teams/noncommuni cable-diseases/surveillance/systems-tools/global-school-based-studenthealth-survey. Accessed 1 Sept 2021.

4. GHDx. STEPS Global School-Based Student Health Survey (GSHS). 2019. Available from: http://ghdx.healthdata.org/series/steps-global-schoolbased-student-health-survey-gshs. Accessed 30 Aug 2021.

5. Abdalmaleki E, Abdi Zh, Ahmadnezhad E, et al. A review of the methodology and tools of childhood \& adolescence surveillance and prevention of adult non-communicable disease survey (CASPIAN) conducted in Iran. Iran J Epidemiol. 2019;15(3):235-46.

6. World Health Organization. STEPwise Approach to NCDs Risk Factor Surveillance (STEPS). Available from: https://www.who.int/teams/nonco mmunicable-diseases/surveillance/systems-tools/steps. Accessed 1 Sept 2021.

7. Center for Disease Control and Prevention Global School-based Student Health Survey (GSHS) Data User's Guide. January. Available from: https:// www.cdc.gov/gshs/background/pdf/gshs-data-users-guide.pdf. Accessed 30 Aug 2021.

8. PAHO. Global School-based Student Health Survey (GSHS). Available from: https://www3.paho.org/hq/index.php?option=com_content\& view=article\&id=13582:gshs\&ltemid=42359\&lang=en\#\&ltemid $=42360$.

9. World Health Organization. GSHS Questionnaire. Access date: 09.01.2021. Available from: https://www.who.int/teams/noncommunicable-diseases/ surveillance/systems-tools/global-school-based-student-health-survey/ questionnaire.

10. Page RM, Danielson M. Multi-country, cross-national comparison of youth tobacco use: findings from global school-based health surveys. Addict Behav. 2011;36(5):470-8.

11. World Health Organization. Global School-based Student Health Survey (GSHS)_Data \& Reporting. Available from: https://www.who.int/teams/ noncommunicable-diseases/surveillance/data. Accessed 8 Mar 2021.

12. World Health Organization, Global school-based student health survey (GSHS) 2004 Core Questionnaire Module Rationale 2004.

13. Abdi Z, Majdzadeh R, Ahmadnezhad E. Developing a framework for the monitoring and evaluation of the Health Transformation Plan in the Islamic Republic of Iran: lessons learned. East Mediterr Health J. 2019;25(6).

14. Lozano R, et al. Measuring progress from 1990 to 2017 and projecting attainment to 2030 of the health-related sustainable development goals for 195 countries and territories: a systematic analysis for the global burden of disease study 2017. Lancet. 2018;392(10159):2091-138.

15. Haghdoost AA, Lari MA, Harirchi I, AhmadnezhadE. Universal Health Coverage Road Map in Eastern Mediterranean Region: Brief Report on Ministerial Meeting, 03-05 September (2018), Salaleh, Oman. Hakim Health Sys Res J. 2018;21(2):147-52.

16. Naik R, Kaneda T. Addressing noncommunicable disease risk factors among young people: Asia's window of opportunity to curb a growing epidemic. Washington, DC: Population Reference Bureau; 2016.

17. Kelishadi $R$, et al. National and sub-national prevalence, trend, and burden of cardiometabolic risk factors in Iranian children and adolescents, 1990-2013 (study protocol). Arch Iran Med. 2014;17(1):71-80. 
18. Kelishadi R, et al. Methodology and early findings of the third survey of CASPIAN study: a national school-based surveillance of students' high risk behaviors. Int J Prev Med. 2012;3(6):394.

19. Kelishadi R, et al. Methodology and early findings of the fourth survey of childhood and adolescence surveillance and prevention of adult non-communicable disease in Iran: the CASPIAN-IV study. Int J Prev Med. 2013;4(12):1451.

20. Motlagh ME, et al. Methodology and early findings of the fifth survey of childhood and adolescence surveillance and prevention of adult noncommunicable disease: the CASPIAN-V study. Int J Prev Med. 2017;8.

21. Kelishadi $R$, et al. An overview of a national surveillance program in Iran for prevention of chronic non-communicable dis-eases from childhood: Caspian study. Iranian J Pub Health. 2009;38(Supple 1):102-6.

22. Khashayar P, et al. Childhood overweight and obesity and associated factors in iranian children and adolescents: a multilevel analysis; the CASPIAN-IV study. Front Pediatr. 2018;6:393.

23. Kelishadi $R$, et al. Thinness, overweight and obesity in a national sample of Iranian children and adolescents: CASPIAN study. Child Care Health Dev. 2008;34(1):44-54.

24. Kelishadi R, et al. Association of physical activity and dietary behaviours in relation to the body mass index in a national sample of Iranian children and adolescents: CASPIAN study. Bull World Health Organ. 2007;85:19-26.

25. Kelishadi $\mathrm{R}$, et al. Smoking behavior and its influencing factors in a national-representative sample of Iranian adolescents: Caspian study. Prev Med. 2006;42(6):423-6.

26. AlBuhairan FS, et al. Time for an adolescent health surveillance system in Saudi Arabia: findings from "Jeeluna". J Adolesc Health. 2015;57(3):263-9.

27. World health organization. Physical activity. 2020; Available from: https:// www.who.int/en/news-room/fact-sheets/detail/physical-activity.

28. World Health Organization. Global action plan for the prevention and control of noncommunicable diseases 2013-2020. World Health Organization; 2013. https://apps.who.int/iris/handle/10665/94384.

29. World Cancer Research Fund International. Why is being physically active important?. Available from: https://www.wcrforg/policy/our-policywork/. Accessed 30 Sept 2021.

30. World Health Organization. Global action plan on physical activity 20182030: more active people for a healthier world. World Health Organization; 2018. https://apps.who.int/iris/handle/10665/272722. License: CC BY-NC-SA 3.0 IGO.

31. Sharara E, et al. Physical inactivity, gender and culture in Arab countries: a systematic assessment of the literature. BMC Public Health. 2018;18(1):1-19.

32. World Health Organization. Tobacco Free initiative. Available from: http:// www.emro.who.int/tfi/what-we-do/index.html. Accessed 30 Oct 2021.

33. World Health Organization. Regional Office for the Eastern Mediterranean. Operationalization of the adolescent health component of the Global Strategy for Women's, Children's and Adolescents' Health, 2016-2030. World Health Organization. Regional Office for the Eastern Mediterranean; 2017. https://apps.who.int/iris/handle/10665/259050.

\section{Publisher's Note}

Springer Nature remains neutral with regard to jurisdictional claims in published maps and institutional affiliations.

Ready to submit your research? Choose BMC and benefit from:

- fast, convenient online submission

- thorough peer review by experienced researchers in your field

- rapid publication on acceptance

- support for research data, including large and complex data types

- gold Open Access which fosters wider collaboration and increased citations

- maximum visibility for your research: over $100 \mathrm{M}$ website views per year

At BMC, research is always in progress.

Learn more biomedcentral.com/submissions 